\title{
The Effect of Combined Patching and Citalopram on Visual Acuity in Adults with Amblyopia: A Randomized, Crossover, Placebo-Controlled Trial
}

\author{
Alice K. Lagas, ${ }^{1}$ Joanna M. Black, ${ }^{1}$ Bruce R. Russell, ${ }^{2}$ Robert R. Kydd, ${ }^{3}$ \\ and Benjamin Thompson $\mathbb{D}^{1,4}$ \\ ${ }^{1}$ School of Optometry and Vision Science, University of Auckland, New Zealand \\ ${ }^{2}$ School of Pharmacy, University of Otago, New Zealand \\ ${ }^{3}$ Department of Psychological Medicine, University of Auckland, New Zealand \\ ${ }^{4}$ School of Optometry and Vision Science, University of Waterlo, Canada
}

Correspondence should be addressed to Benjamin Thompson; ben.thompson@uwaterloo.ca

Received 25 March 2019; Accepted 14 May 2019; Published 9 June 2019

Academic Editor: Malgorzata Kossut

Copyright ( $) 2019$ Alice K. Lagas et al. This is an open access article distributed under the Creative Commons Attribution License, which permits unrestricted use, distribution, and reproduction in any medium, provided the original work is properly cited.

\begin{abstract}
Nonhuman animal models have demonstrated that selective serotonin reuptake inhibitors (SSRIs) can enhance plasticity within the mature visual cortex and enable recovery from amblyopia. The aim of this study was to test the hypothesis that the SSRI citalopram combined with part-time patching of the fellow fixing eye would improve amblyopic eye visual acuity in adult humans. Following a crossover, randomized, double-blind, placebo-controlled design, participants completed two 2-week blocks of fellow fixing eye patching. One block combined patching with citalopram $(20 \mathrm{mg} /$ day $)$ and the other with a placebo tablet. The blocks were separated by a 2 -week washout period. The primary outcome was change in amblyopic eye visual acuity. Secondary outcomes included stereoacuity and electrophysiological measures of retinal and cortical function. Seven participants were randomized, fewer than our prespecified sample size of 20 . There were no statistically significant differences in amblyopic eye visual acuity change between the active (mean \pm SD change $=0.08 \pm 0.16 \log$ MAR) and the placebo (mean change $=-0.01 \pm 0.03 \log$ MAR) blocks. No treatment effects were observed for any secondary outcomes. However, 3 of 7 participants experienced a $0.1 \log$ MAR or greater improvement in amblyopic eye visual acuity in the active but not the placebo blocks. These results from a small sample suggest that larger-scale trials of SSRI treatment for adult amblyopia may be warranted. Considerations for future trials include drug dose, treatment duration, and recruitment challenges. This study was preregistered as a clinical trial (ACTRN12611000669998).
\end{abstract}

\section{Introduction}

Disruptions to binocular vision such as strabismus (an eye turn) or anisometropia (unequal refractive error between the two eyes) during the critical period of visual development can cause a neurodevelopmental disorder of vision called amblyopia $[1,2]$. The deficits associated with amblyopia encompass a wide range of monocular and binocular visual functions [3, 4] and also extend to the fellow fixing eye [5]. Clinically, amblyopia is typically diagnosed on the basis of a monocular visual acuity loss that cannot be explained by ocular pathology combined with an amblyogenic factor [1]. Cur- rent treatments for amblyopia in childhood involve the provision of refractive correction followed by patching or penalization of the fellow fixing eye to promote use of the amblyopic eye. These treatments are effective [6-12], but efficacy appears to decline with increasing age in children [13-16], possibly due to a decline in neural plasticity as the visual cortex matures and exits the critical period for visual development [17-20]. A growing body of literature demonstrates that vision can improve in adult humans with amblyopia through interventions such as monocular [21, 22] and binocular [23-27] perceptual learning and noninvasive brain stimulation [28-32]. However, these approaches have 


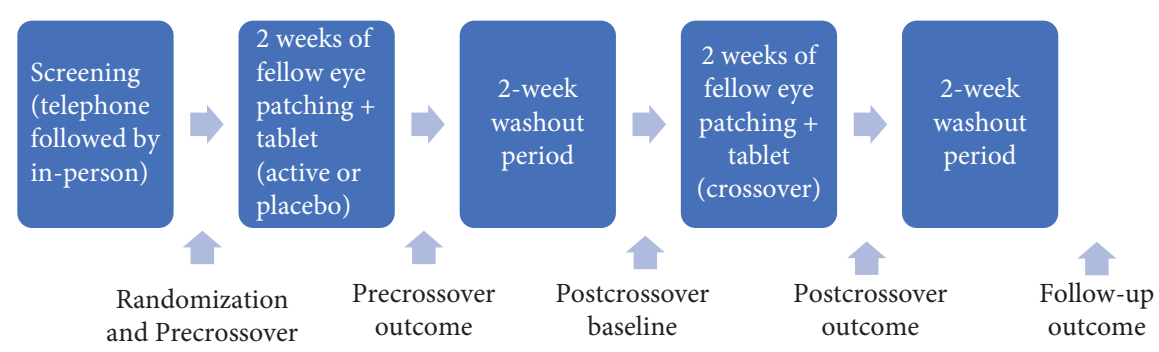

FIgURE 1: Schematic of the study protocol and the timing of baseline, outcome, and follow-up measures.

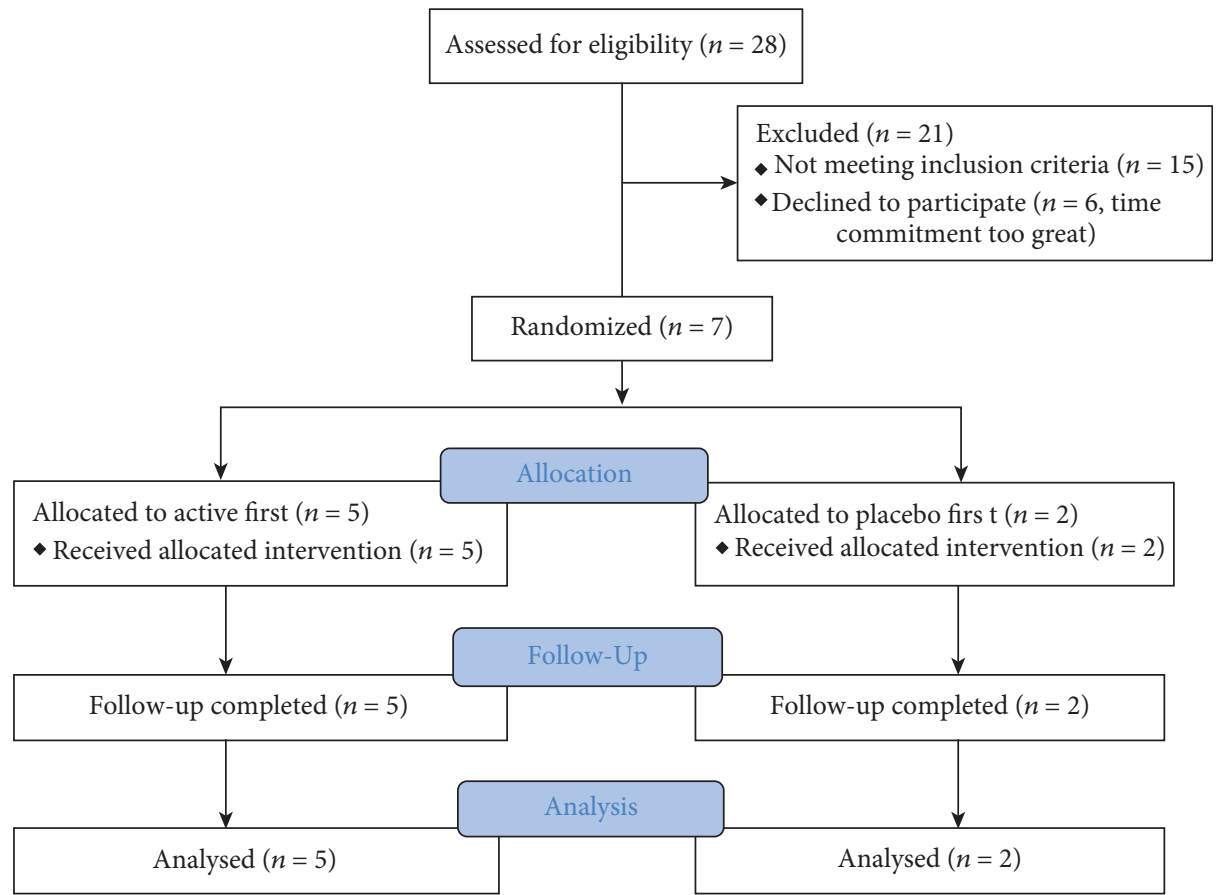

FIgURE 2: CONSORT diagram for the study.

not yet translated into positive randomized clinical trials in adult patients that are required for evidence-based clinical practice [33].

Amblyopia also forms the basis of a prominent nonhuman animal model for studying cortical development and plasticity [34]. Monocular amblyopia can be induced in nonhuman animals within the critical period of visual development using an eyelid suture, induction of strabismus, or provision of anisometropic refractive error [35]. Over the past decade or so, a considerable number of studies have used this model to explore postcritical period neuroplasticity [36]. Successful interventions for amblyopia recovery in postcritical period animal models include dark exposure $[37,38]$, enriched visual environments [39], food restriction [40], binocular training [41], physical exercise [42], and retinal inactivation [43].

Pharmaceutical interventions have also been investigated in rodent models of amblyopia. A particularly striking result was reported by Vetencourt et al. [44] whereby chronic administration of the selective serotonin reuptake inhibitor (SSRI) fluoxetine enabled recovery of normal visual cortex responses and visual acuity in mature rats with unilateral deprivation amblyopia. This effect occurred when fluoxetine was administered before and during eyelid suture of the nondeprived eye and opening of the deprived eye (a procedure known as a reverse suture). The improvements in visual function were linked to reduced GABA-mediated inhibition within the visual cortex and increased expression of brainderived neurotrophic factor (BDNF). This finding is of particular interest in the context of amblyopia treatment in adult humans because SSRIs are widely available to clinicians. Furthermore, SSRIs may enhance plasticity within the human motor [45, 46] and visual [47] cortexes. Fluoxetine has also been found to enhance physiotherapy outcomes after stroke, possibly by increasing cortical plasticity [48]. However, fluoxetine did not enhance visual perceptual learning of a motion discrimination task or motor cortex plasticity in a study of healthy human adults [49].

Two studies have investigated the use of fluoxetine to treat human amblyopia. Sharif et al. [50] compared 3 months of fellow fixing eye patching plus fluoxetine $(0.5 \mathrm{mg} / \mathrm{kg} / \mathrm{day}$, $n=20)$ to patching plus a placebo tablet placebo $(n=15)$ in 


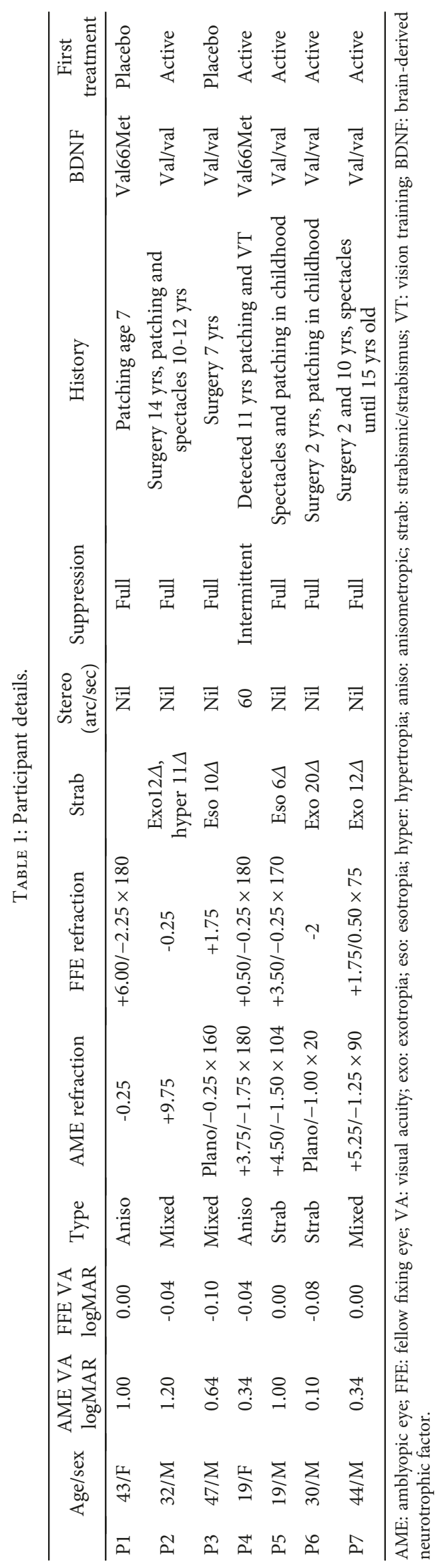


TABLE 2: Amblyopic eye visual acuity results.

\begin{tabular}{|c|c|c|c|c|c|c|c|}
\hline & Active baseline & Active outcome & Active change & Placebo baseline & Placebo outcome & Placebo change & Final washout \\
\hline $\mathrm{P} 1$ & 0.94 & 0.82 & 0.12 & 1 & 0.97 & 0.03 & 0.87 \\
\hline P2 & 1.20 & 1.20 & 0.00 & 1 & 1 & 0 & 1.1 \\
\hline P3 & 0.73 & 0.60 & 0.13 & 0.64 & 0.67 & -0.03 & 0.633 \\
\hline P4 & 0.34 & 0.36 & -0.02 & 0.32 & 0.32 & 0 & 0.3 \\
\hline P5 & 1.00 & 0.60 & 0.40 & 0.866 & 0.9 & -0.034 & 0.74 \\
\hline P6 & 0.10 & 0.10 & 0.00 & 0.1 & 0.08 & 0.02 & 0.14 \\
\hline P7 & 0.34 & 0.40 & -0.06 & 0.32 & 0.36 & -0.04 & 0.32 \\
\hline Mean (SD) & $0.66(0.36)$ & $0.58(0.35)$ & $0.08(0.16)$ & $0.61(0.36)$ & $0.61(0.36)$ & $-0.01(0.03)$ & $0.59(0.35)$ \\
\hline
\end{tabular}

Change values were calculated by subtracting the outcome from the baseline. All values are in logMAR.

TABLE 3: Self-reported patching adherence data sourced from participants' patching diaries.

\begin{tabular}{lccc}
\hline & Active & Placebo & Difference \\
\hline P1 & $116(31)$ & $114(24)$ & 2 \\
P2 & $40(19)$ & $29(21)$ & 11 \\
P3 & $55(28)$ & $55(7)$ & 1 \\
P4 & $96(38)$ & $111(32)$ & -15 \\
P5 & $75(0)$ & $75(0)$ & 0 \\
P6 & $111(78)$ & $111(74)$ & 0 \\
P7 & $111(32)$ & $111(32)$ & 0 \\
Mean (SD) & $\mathbf{8 6 ( 3 0 )}$ & $\mathbf{8 7 ( 3 4 )}$ & $\mathbf{0 . 1 ( 7 . 6 )}$ \\
\hline
\end{tabular}

Data are shown as mean minutes of patching per day (SD). The prescribed dose was 120 minutes per day.

older children and adults (10-40 years) with amblyopia. A significantly greater amblyopic eye visual acuity improvement in the fluoxetine compared to the placebo group was observed. However, Huttunen et al. [51] found no differences in visual function improvement between a group of adults with amblyopia treated for 10 days with combined perceptual learning and fluoxetine (20 mg per day, $n=22$ ) and a group treated with perceptual learning combined with a placebo tablet $(n=20)$.

In this study, we explored the effects of 2 weeks (14 days) of the SSRI citalopram combined with fellow fixing eye patching on visual acuity, stereopsis, and visually evoked retinal and cortical responses in adults with amblyopia. We anticipated that recruitment would be challenging due to the use of patching and the administration of an antidepressant drug. We therefore adopted a placebo-controlled, randomized, double-blind, crossover design. In this context, citalopram was chosen over fluoxetine (as used in prior studies) because citalopram has a shorter half-life [52] that allowed for a manageable washout period to be incorporated into the design of the study. No significant effects of citalopram were observed, although our study may have been underpowered due to recruitment challenges.

\section{Methods}

2.1. Trial Design. The single-site trial involved two blocks of fellow fixing eye patching each lasting two weeks separated by a two-week washout period. Participants were provided with citalopram $(1 \times 20 \mathrm{mg}$ tablet per day) during one patching block and otherwise identical placebo tablets (sucrose) during the other block. Block order was randomized using a random number generator. The timing of the baseline and the outcome measures are shown in Figure 1. Only the pharmacist dispensing the tablets, who did not interact with study participants, was unmasked to block order. Study participants and all other members of the research team were masked to the randomization. The study was approved by the Northern X Regional Ethics Committee in New Zealand (NTX/11/06/044) and preregistered as a clinical trial (ACTRN12611000669998).

Participants completed a screening protocol consisting of a telephone interview followed by a full optometric examination, medical history, the Profile of Mood States Short Form (POMS-SF) questionnaire, and the Depression Anxiety and Stress Scale (DASS-21).

Study inclusion criteria were as follows: 18 years of age or over, $0.2 \log$ MAR or worse visual acuity in the amblyopic eye, $0.0 \log$ MAR or better visual acuity in the fellow fixing eye, an interocular acuity difference of at least $0.2 \log$ MAR, and the presence of a strabismus and/or anisometropia defined as a difference in spherical equivalent refractive error of 1.5 dioptres or greater between the eyes. The exclusion criteria were as follows: the presence of ocular pathology, an explanation for the visual acuity loss other than amblyopia, personal or family history of a mood disorder, diabetes, history of addiction, current use of medications or supplements known to alter mood, medications that interact with SSRIs such as codeine, and abnormal mood states evident on the mood questionnaires as reviewed by a psychiatrist. Prior to randomization, participants who were not wearing optimal full correction for both eyes were provided with full correction (either spectacles or contact lenses) and were reviewed every four weeks until visual acuity was stable $(<0.2 \operatorname{logMAR}$ difference between visits). Participants were recruited through the University of Auckland Optometry Clinic, referral from eye care practitioners, word of mouth, and newspaper advertisements. Participants were compensated for their time.

2.2. Baseline and Outcome Measures. Visual acuity (VA) was assessed using a computerized ETDRS chart (Medmont) from $6 \mathrm{~m}$. The right eye was tested first. Each correctly 


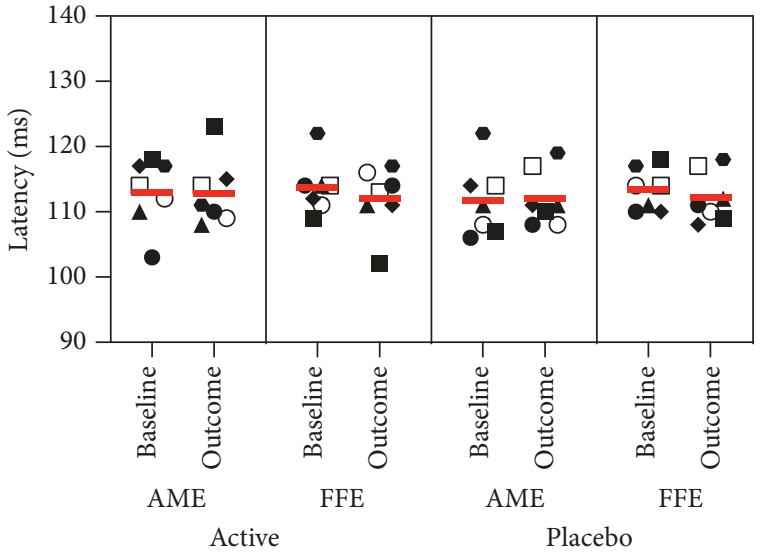

(a)

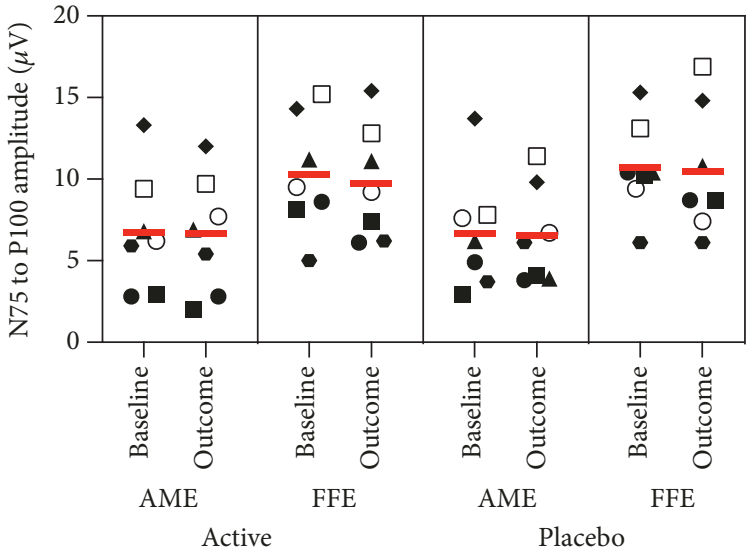

(b)

Figure 3: VEP results for the $1^{\circ}$ check stimulus. Latencies for the P100 component are shown in (a), and amplitudes for the N57-P100 waveform component are in (b). Individual participants are shown with different symbols-P1-7 as follows: filled circle, filled square, filled triangle, filled diamond, filled hexagon, open circle, and open square, respectively. Horizontal lines depict group mean values.

identified letter was worth $0.02 \log$ MAR. Binocular vision was assessed using a unilateral cover test, a prism cover test, the Worth 4-dot test $(33 \mathrm{~cm}$ and $6 \mathrm{~m})$, and the TNO stereoacuity test. Electrophysiological measurements of retinal and visual cortex function were made using ISCEVstandardized electrophysiological protocols on a Roland RETIscan system (software version 4.13.1.8). The following tests were applied monocularly (right eye first): pattern ERG $\left(1^{\circ}\right.$ check size-modified from the $0.8^{\circ}$ standard for direct comparison with the VEP stimuli), VEP $\left(1^{\circ}\right.$ and $0.3^{\circ}$ check sizes), and multifocal ERG with pupil dilation. ERG measures were included so that any retinal effects of citalopram could be accounted for if the trial was positive. The POMS-SF questionnaire was completed at each study visit, and participants completed a patching diary for each 2-week patching session. The brain-derived neurotrophic factor (BNDF) phenotype has been identified as a possible mediator of cortical plasticity [53], and BNDF upregulation has been identified as a mechanism for increased visual cortex plasticity following fluoxetine administration in rats [44]. To test for BDNF polymorphisms, participants provided a blood sample directly after the first two-week block of patching. Following a previously reported protocol [49], an Agena MassARRAY iPLEX assay (Agena Bioscience, San Diego, CA, USA) was used for genotyping. A Bruker Mass Spectrometer with optimized parameters for iPLEX chemistry was then used to resolve single base extensions. Typer 4 analysis software (Agena Bioscience) enabled visual inspection of generated peaks in comparison to the nontemplate control.

2.3. Statistical Analysis. At the time of study initiation, no previous studies of SSRIs in human amblyopia treatment were available. Therefore, we selected a sample size of 20 based on recruitment estimates for the study site. Outcome measures were analysed separately using mixed ANOVAs with within-subject factors of Session (baseline vs. outcome) and Treatment (active vs. placebo) and a between-subject factor of Group (active first vs. placebo first).

\section{Results}

Sixty-one participants expressed interest in the study and were sent a study information package. Twenty-eight participants responded and were assessed for eligibility. Seven participants were randomized. The CONSORT diagram for these participants is shown in Figure 2. Reasons for exclusion included time commitment too great, medical or recreational use of drugs, vision too good in the amblyopic eye, and diabetes. One participant who did not meet the visual acuity inclusion criteria was randomized (P6, see Table 1). Data from this participant were included in the final analysis due to the small sample size. Randomized participant details, including BDNF polymorphism, are shown in Table 1.

Baseline and outcome data for amblyopic eye visual acuity are shown in Table 2 . There was no significant interaction between the Session and Treatment factors $\left(F_{1,5}=1.7, p=\right.$ 0.25 , and partial $\left.\eta^{2}=0.26\right)$ indicating no difference between the active and the placebo treatment. The Session factor also had no main effect indicating the absence of a visual acuity improvement across the two periods of patching $\left(F_{1,5}=1.7\right.$, $p=0.25$, and partial $\eta^{2}=0.26$ ). Overall, no main effects or interactions were significant in the analysis (all $p>0.25$ ). An inspection of individual data (Table 2) indicated that $3 / 7$ participants improved by $>0.1 \log$ MAR in the active but not the placebo condition. One of these participants had a val66met BDNF polymorphism. The remaining two had $\mathrm{val} / \mathrm{val}$ BNDF polymorphisms. No main effects or interactions were present for the fellow fixing eye visual acuity data (all $F<3.9$, all $p>0.1$, and all partial $\eta^{2}<0.4$ ).

Adherence data are shown in Table 3. Adherence did not differ significantly between active and placebo blocks $\left(t_{6}=1.0, p=0.9\right)$. On average, participants had approximately $70 \%$ adherence with the 120 minutes per day of prescribed patching. There was no correlation between patching adherence and visual acuity change in either the active $\left(r_{7}=-0.2, p=0.6\right)$ or placebo $\left(r_{7}=0.3, p=0.5\right)$ blocks. 

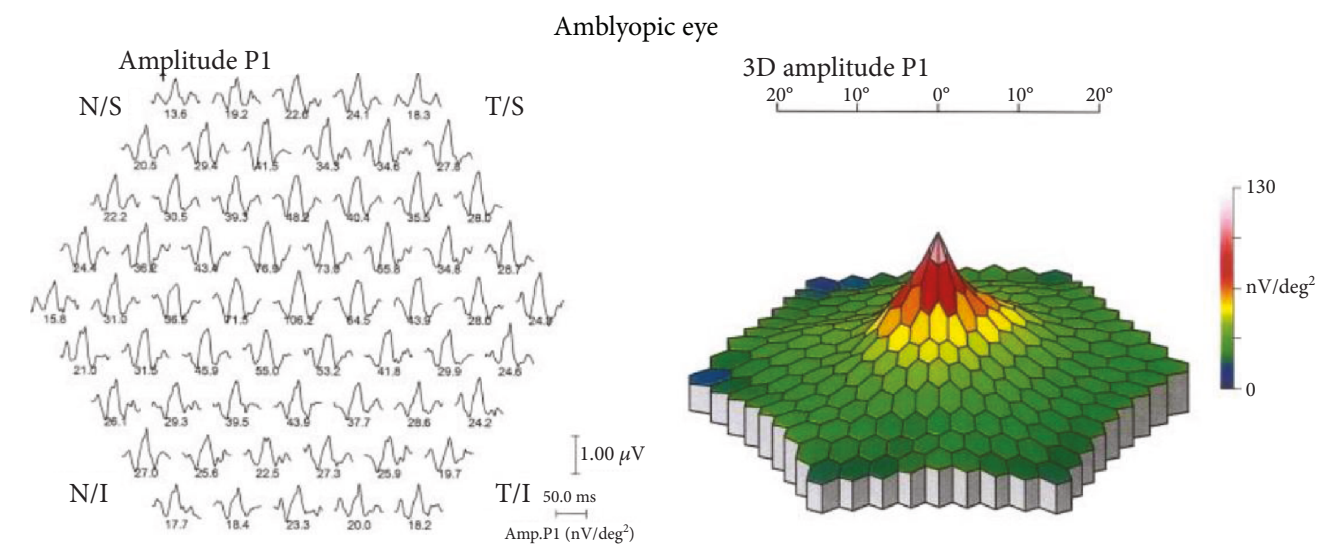

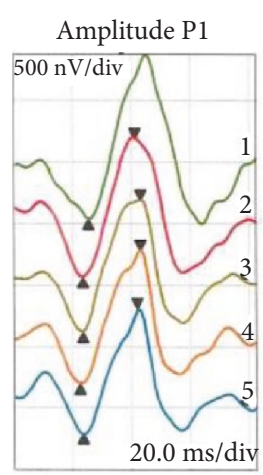

Amplitude P1

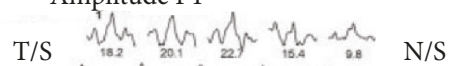

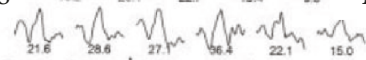

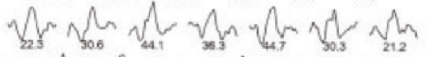

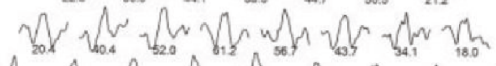

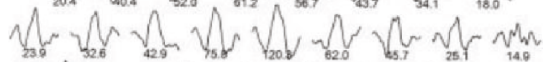

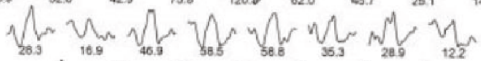

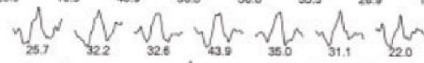

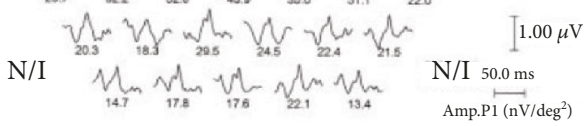

\begin{tabular}{ccccc}
\hline Ring & $\begin{array}{c}\text { Amp.P1 } \\
\left(\mathrm{nV} / \mathrm{deg}^{2}\right)\end{array}$ & Normal & $\begin{array}{c}\text { PeT.P1 } \\
(\mathrm{ms})\end{array}$ & $\begin{array}{c}\text { Ratio } \\
(\mathrm{R} 1 / \mathrm{Rx})\end{array}$ \\
\hline 1 & 106.2 & $66.6-130.8$ & 45.1 & 1 \\
2 & 63.2 & $30.9-77.8$ & 41.2 & 1.68 \\
3 & 41.2 & $21.7-59$ & 43.1 & 2.58 \\
4 & 30.1 & $12.9-37.1$ & 43.1 & 3.53 \\
5 & 21.1 & $10-28.2$ & 42.1 & 5.02 \\
\hline
\end{tabular}

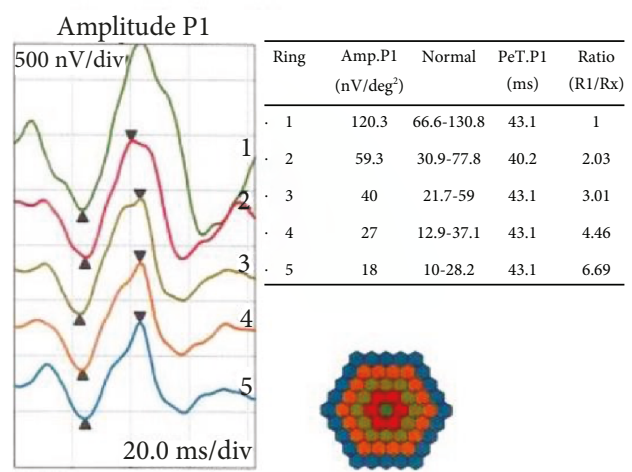

Fellow eye
Amplitude P1
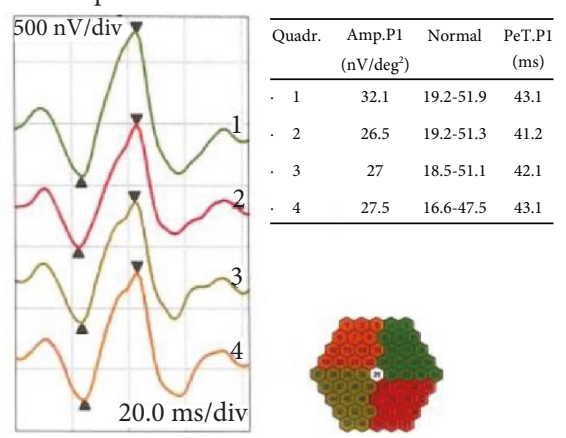

(a)
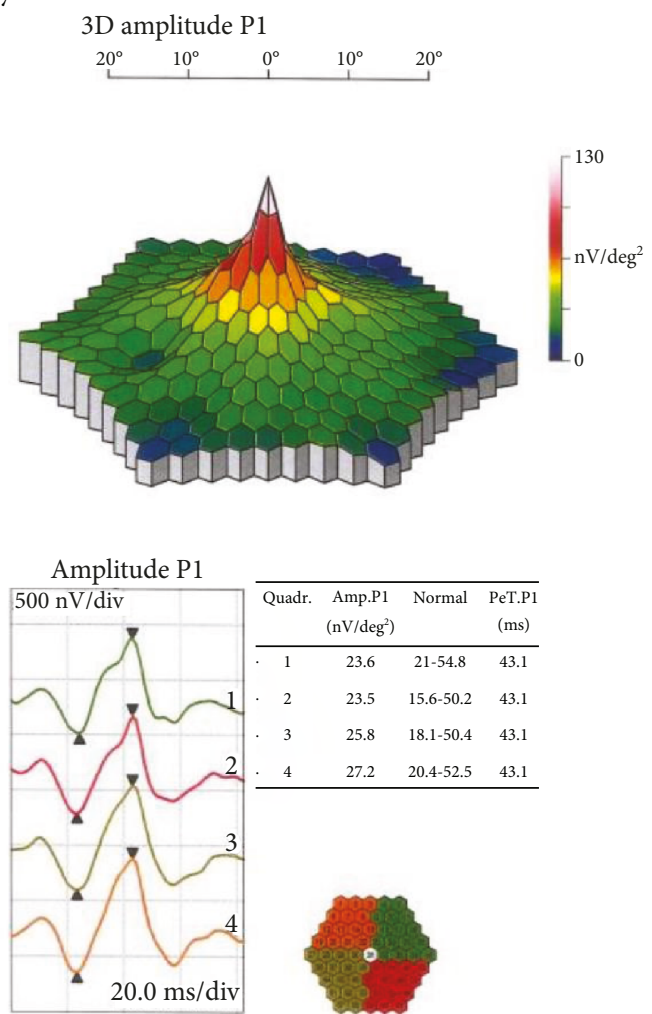

(b)

FIgURE 4: Multifocal ERG results for participant P7 (first baseline session). 


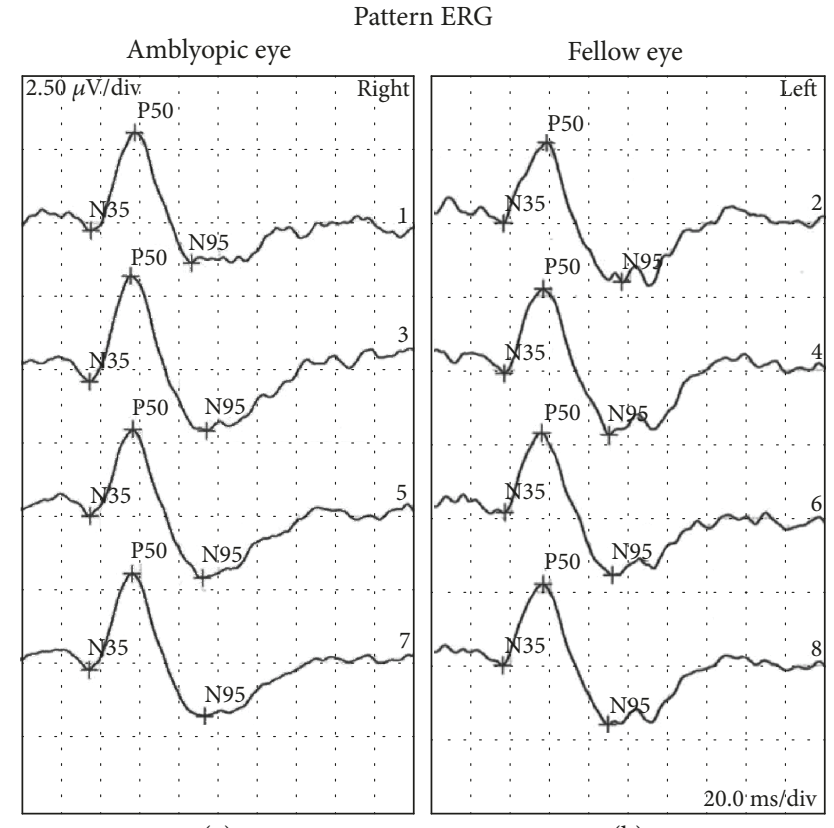

(a)

(b)

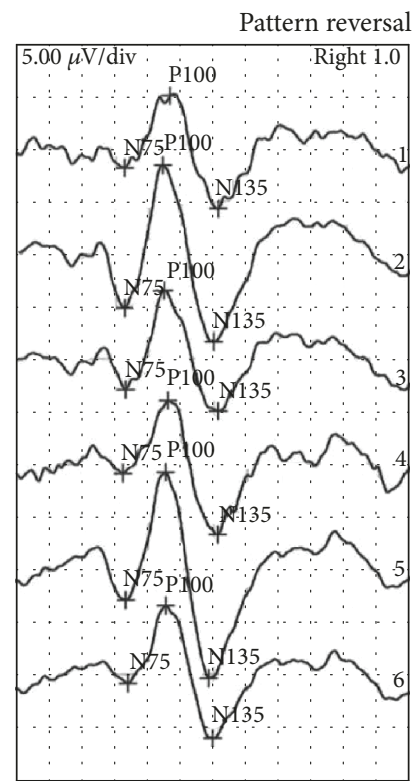

(c)

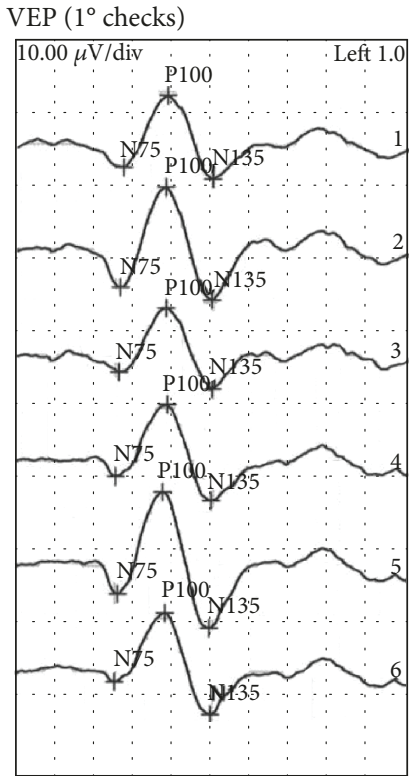

(d)
Figure 5: Pattern ERG ( $a, b)$ and pattern reversal VEP (c, d) results for the amblyopic $(\mathrm{a}, \mathrm{c})$ and fellow $(\mathrm{b}, \mathrm{d})$ eyes of participant P7 (first baseline session).

Only participant P6 exhibited a change in stereoacuity, improving from nil to $240 \mathrm{arc} / \mathrm{sec}$ in the active block and from nil to $480 \mathrm{arc} / \mathrm{sec}$ in the placebo block. Follow-up stereoacuity was nil. No significant treatment effects were evident for any of the electrophysiological measurements (all $F<2.0$, all $p>2$ ). Figure 3 shows $1^{\circ}$ check stimulus VEP latencies (left) and N75-P100 amplitudes (right) for both the amblyopic and fellow fixing eyes. Figure 4 shows example multifocal ERG data for participant P7 (first baseline measure), and Figure 5 shows example pattern ERG and VEP data for the same participant. There were no treatment effects on POM-SF scores.

\section{Discussion}

The SSRI fluoxetine enabled recovery of vision in mature rats with amblyopia [44] and has recently been reported to enhance the effect of patching in older children and adults [50]. We found no effect of the SSRI citalopram combined with two weeks of patching on amblyopic eye visual acuity or a range of secondary outcome measures in adults with amblyopia. These results are broadly consistent with another recent study with a similar duration treatment period (10 days) that reported no advantage of combining fluoxetine with perceptual learning compared to perceptual learning alone in adults with amblyopia [51]. A preliminary study of donepezil [54] and a randomized clinical trial of levodopa [55] have also found no benefit of drug treatment in amblyopia therapy. In addition, we found no effect of two weeks of patching alone in adult patients despite reasonable selfreported adherence. This is expected based on the short treatment period and the reduced effect of patching with increasing age $[15,16,56]$.

A number of factors may explain the lack of a drug treatment effect in our study. First, and perhaps most importantly, we did not achieve our planned sample size of 20 participants due to difficulties with recruitment. This led to a small sample with varied amblyopia etiology and treatment history. Barriers to recruitment included the time commitment required by the study and the stringent medical inclusion criteria. Therefore, our study may be underpowered to detect a treatment effect, although the sample size is within the range of previous case-series perceptual learning studies that have reported treatment effects [57]. It is intriguing that three participants exhibited an amblyopic eye visual acuity improvement of $0.1 \operatorname{logMAR}$ or greater for the active but not the placebo treatment sessions. These participants had relatively poor baseline amblyopic eye visual acuity compared to most of the other participants. No participants exhibited any improvement for the placebo sessions. This observation suggests that further testing of SSRI treatment effects in adults with amblyopic may be warranted.

Whereas previous studies have used fluoxetine, we used citalopram because it has a short lead-in period of two hours [58]. Moreover, citalopram has a shorter half-life than fluoxetine; the distribution phase lasts about ten hours and the terminal half-life (T1/2) is 30-35 hours for citalopram [58] in contrast to two to four days' half-life for fluoxetine [59]. Citalopram and fluoxetine appear to have the same efficacy for treating major depression [60] and comparable effects on plasma GABA, glutamine, and glutamate levels in human patients [61]. However, citalopram and fluoxetine have different patterns of binding affinity within the human brain [62]. It is currently unknown whether the two drugs differ in the extent to which they promote visual cortex plasticity.

We used a $20 \mathrm{mg} /$ day dose of citalopram over 2 weeks. It is possible that larger doses and longer treatment times are required to replicate the effects found in nonhuman animals. Supporting this idea, Sharif et al. [50] found a significant 
effect of combined fluoxetine and patching with a dose of 0.5 $\mathrm{mg} / \mathrm{kg} /$ day and a 3-month treatment period whereas Huttunen et al. [51] found no effect with $20 \mathrm{mg}$ per day over 10 days. The parameter space for dosing and treatment duration is large for drug intervention studies of this type, and further work is required to identify optimal values. In addition, genotype may also influence an individual's response to a pharmacological intervention. In this study, we measured BDNF polymorphisms because they have been linked to neuroplasticity [53] and an increase in BDNF expression has been identified as a key mechanism in SSRI-induced recovery from amblyopia in mature rats [44]. There was no relationship between BDNF polymorphism and treatment response in this study with both val/val and val66met carriers improving by $1 \log$ MAR line or more. However, the small sample size precludes any strong conclusions.

In agreement with Huttunen et al. [51], we found no effect of SSRI treatment on VEP parameters. This is in contrast to other emerging potential approaches to amblyopia treatment in adulthood such as the noninvasive brain stimulation technique anodal transcranial direct current stimulation that increases VEP amplitude [28]. The lack of any VEP changes is consistent with the lack of a treatment effect on any of the other outcome measures used within this study. Retinal electrophysiology was also conducted to rule out any retinal changes if a positive treatment effect was observed. No retinal changes were observed, in agreement with the overall study results.

In addition to the small sample size, a weakness of our study is that one participant (P6) did not meet the visual acuity inclusion criteria. We retained this participant in the study due to difficulties with recruitment. We note that excluding this participant from the sample does not change the pattern of results.

In conclusion, we found no effect of 2 weeks of combined citalopram and patching on amblyopic eye visual acuity in adults with amblyopia. This result may have been due to our study being underpowered as a result of recruitment challenges. Three out of seven participants did exhibit an amblyopic eye visual acuity improvement of $0.1 \log$ MAR or more with combined citalopram and patching suggesting that further studies in this area may be warranted.

\section{Data Availability}

All clinical data are provided within the manuscript tables. Anonymized electrophysiological data are available from the authors upon request.

\section{Conflicts of Interest}

The authors have no conflicts of interest to declare.

\section{Acknowledgments}

This study was supported by grants to BT from the Marsden Fund of New Zealand and the Canadian Institutes of Health Research (CIHR 365136).

\section{References}

[1] J. M. Holmes and M. P. Clarke, "Amblyopia," The Lancet, vol. 367, no. 9519, pp. 1343-1351, 2006.

[2] D. Maurer and K. S. Mc, "Classification and diversity of amblyopia," Visual Neuroscience, vol. 35, article E012, 2018.

[3] L. M. Hamm, J. Black, S. Dai, and B. Thompson, "Global processing in amblyopia: a review," Frontiers in Psychology, vol. 5, p. 583, 2014.

[4] A. M. Wong, "New concepts concerning the neural mechanisms of amblyopia and their clinical implications," Canadian Journal of Ophthalmology, vol. 47, no. 5, pp. 399409, 2012.

[5] K. Meier and D. Giaschi, "Unilateral amblyopia affects two eyes: fellow eye deficits in amblyopia," Investigative Opthalmology \& Visual Science, vol. 58, no. 3, pp. 1779-1800, 2017.

[6] J. M. Holmes, "Designing clinical trials for amblyopia," Vision Research, vol. 114, pp. 41-47, 2015.

[7] C. E. Stewart, M. J. Moseley, D. A. Stephens, and A. R. Fielder, "Treatment dose-response in amblyopia therapy: the Monitored Occlusion Treatment of Amblyopia Study (MOTAS)," Investigative Opthalmology \& Visual Science, vol. 45, no. 9, pp. 3048-3054, 2004.

[8] Pediatric Eye Disease Investigator Group Writing, “A randomized trial comparing Bangerter filters and patching for the treatment of moderate amblyopia in children," Ophthalmology, vol. 117, no. 5, pp. 998-1004.e6, 2010.

[9] M. X. Repka and J. M. Holmes, "Lessons from the amblyopia treatment studies," Ophthalmology, vol. 119, no. 4, pp. 657658, 2012.

[10] M. M. Scheiman, R. W. Hertle, R. W. Beck et al., "Randomized trial of treatment of amblyopia in children aged 7 to 17 years," Archives of Ophthalmology, vol. 123, no. 4, pp. 437-447, 2005.

[11] Pediatric Eye Disease Investigator Group, "Patching vs atropine to treat amblyopia in children aged 7 to 12 years: a randomized trial," Archives of Ophthalmology, vol. 126, no. 12, pp. 1634-1642, 2008.

[12] D. K. Wallace and Pediatric Eye Disease Investigator Group, "A randomized trial to evaluate 2 hours of daily patching for strabismic and anisometropic amblyopia in children," Ophthalmology, vol. 113, no. 6, pp. 904-912, 2006.

[13] C. E. Stewart, D. A. Stephens, A. R. Fielder, M. J. Moseley, and M. Cooperative, "Modeling dose-response in amblyopia: toward a child-specific treatment plan," Investigative Opthalmology \& Visual Science, vol. 48, no. 6, pp. 2589-2594, 2007.

[14] J. M. Holmes and D. M. Levi, "Treatment of amblyopia as a function of age," Visual Neuroscience, vol. 35, article E015, 2018.

[15] J. M. Holmes, E. L. Lazar, B. M. Melia et al., "Effect of age on response to amblyopia treatment in children," Archives of Ophthalmology, vol. 129, no. 11, pp. 1451-1457, 2011.

[16] M. Fronius, L. Cirina, H. Ackermann, T. Kohnen, and C. M. Diehl, "Efficiency of electronically monitored amblyopia treatment between 5 and 16 years of age: new insight into declining susceptibility of the visual system," Vision Research, vol. 103, pp. 11-19, 2014.

[17] C. Blakemore and R. C. Van Sluyters, "Reversal of the physiological effects of monocular deprivation in kittens: further evidence for a sensitive period," The Journal of Physiology, vol. 237, no. 1, pp. 195-216, 1974. 
[18] D. H. Hubel and T. N. Wiesel, "The period of susceptibility to the physiological effects of unilateral eye closure in kittens," The Journal of Physiology, vol. 206, no. 2, pp. 419-436, 1970.

[19] J. A. Movshon, "Reversal of the physiological effects of monocular deprivation in the kitten's visual cortex," The Journal of Physiology, vol. 261, no. 1, pp. 125-174, 1976.

[20] N. W. Daw, Visual development, Springer, New York, NY, USA, 3rd edition edition, 2014.

[21] D. M. Levi, "Perceptual learning in adults with amblyopia: a reevaluation of critical periods in human vision," Developmental Psychobiology, vol. 46, no. 3, pp. 222-232, 2005.

[22] D. M. Levi and R. W. Li, "Perceptual learning as a potential treatment for amblyopia: a mini-review," Vision Research, vol. 49, no. 21, pp. 2535-2549, 2009.

[23] R. F. Hess and B. Thompson, "Amblyopia and the binocular approach to its therapy," Vision Research, vol. 114, pp. 4-16, 2015.

[24] R. F. Hess, B. Thompson, and D. H. Baker, "Binocular vision in amblyopia: structure, suppression and plasticity," Ophthalmic and Physiological Optics, vol. 34, no. 2, pp. 146-162, 2014.

[25] J. Li, D. P. Spiegel, R. F. Hess et al., "Dichoptic training improves contrast sensitivity in adults with amblyopia," Vision Research, vol. 114, pp. 161-172, 2015.

[26] J. Li, B. Thompson, D. Deng, L. Y. Chan, M. Yu, and R. F. Hess, "Dichoptic training enables the adult amblyopic brain to learn," Current Biology, vol. 23, no. 8, pp. R308-R309, 2013.

[27] I. Vedamurthy, M. Nahum, S. J. Huang et al., "A dichoptic custom-made action video game as a treatment for adult amblyopia," Vision Research, vol. 114, pp. 173-187, 2015.

[28] Z. Ding, J. Li, D. P. Spiegel et al., "The effect of transcranial direct current stimulation on contrast sensitivity and visual evoked potential amplitude in adults with amblyopia," Scientific Reports, vol. 6, no. 1, article 19280, 2016.

[29] B. Moret, R. Camilleri, A. Pavan et al., "Differential effects of high-frequency transcranial random noise stimulation (hftRNS) on contrast sensitivity and visual acuity when combined with a short perceptual training in adults with amblyopia," Neuropsychologia, vol. 114, pp. 125-133, 2018.

[30] D. P. Spiegel, W. D. Byblow, R. F. Hess, and B. Thompson, "Anodal transcranial direct current stimulation transiently improves contrast sensitivity and normalizes visual cortex activation in individuals with amblyopia," Neurorehabil Neural Repair, vol. 27, no. 8, pp. 760-769, 2013.

[31] D. P. Spiegel, J. Li, R. F. Hess et al., "Transcranial direct current stimulation enhances recovery of stereopsis in adults with amblyopia," Neurotherapeutics, vol. 10, no. 4, pp. 831-839, 2013.

[32] B. Thompson, B. Mansouri, L. Koski, and R. F. Hess, "Brain plasticity in the adult: modulation of function in amblyopia with rTMS," Current Biology, vol. 18, no. 14, pp. 1067-1071, 2008.

[33] T. Y. Gao, C. X. Guo, R. J. Babu et al., "Effectiveness of a binocular video game vs placebo video game for improving visual functions in older children, teenagers, and adults with amblyopia: a randomized clinical trial," JAMA Ophthalmology, vol. 136, no. 2, pp. 172-181, 2018.

[34] H. Morishita and T. K. Hensch, "Critical period revisited: impact on vision," Current Opinion in Neurobiology, vol. 18, no. 1, pp. 101-107, 2008.
[35] D. Mitchell and F. Sengpiel, "Animal models of amblyopia," Visual Neuroscience, vol. 35, article E017, 2018.

[36] M. P. Stryker and S. Löwel, “Amblyopia: new molecular/pharmacological and environmental approaches," Visual Neuroscience, vol. 35, article E018, 2018.

[37] H. Y. He, B. Ray, K. Dennis, and E. M. Quinlan, "Experiencedependent recovery of vision following chronic deprivation amblyopia," Nature Neuroscience, vol. 10, no. 9, pp. 11341136, 2007.

[38] K. R. Duffy and D. E. Mitchell, "Darkness alters maturation of visual cortex and promotes fast recovery from monocular deprivation," Current Biology, vol. 23, no. 5, pp. 382-386, 2013.

[39] A. Sale, J. F. Maya Vetencourt, P. Medini et al., "Environmental enrichment in adulthood promotes amblyopia recovery through a reduction of intracortical inhibition," Nature Neuroscience, vol. 10, no. 6, pp. 679-681, 2007.

[40] M. Spolidoro, L. Baroncelli, E. Putignano, J. F. Maya-Vetencourt, A. Viegi, and L. Maffei, "Food restriction enhances visual cortex plasticity in adulthood," Nature Communications, vol. 2, no. 1, p. 320, 2011.

[41] K. M. Murphy, G. Roumeliotis, K. Williams, B. R. Beston, and D. G. Jones, "Binocular visual training to promote recovery from monocular deprivation," Journal of Vision, vol. 15, no. 1, p. 2, 2015.

[42] M. Kaneko and M. P. Stryker, "Sensory experience during locomotion promotes recovery of function in adult visual cortex," Elife, vol. 3, article e02798, 2014.

[43] M. F. Fong, D. E. Mitchell, K. R. Duffy, and M. F. Bear, "Rapid recovery from the effects of early monocular deprivation is enabled by temporary inactivation of the retinas," Proceedings of the National Academy of Sciences of the United States of America, vol. 113, no. 49, pp. 14139-14144, 2016.

[44] J. F. Maya Vetencourt, A. Sale, A. Viegi et al., "The antidepressant fluoxetine restores plasticity in the adult visual cortex," Science, vol. 320, no. 5874, pp. 385-388, 2008.

[45] I. Loubinoux, J. Pariente, O. Rascol, P. Celsis, and F. Chollet, "Selective serotonin reuptake inhibitor paroxetine modulates motor behavior through practice. A double-blind, placebocontrolled, multi-dose study in healthy subjects," Neuropsychologia, vol. 40, no. 11, pp. 1815-1821, 2002.

[46] I. Loubinoux, D. Tombari, J. Pariente et al., "Modulation of behavior and cortical motor activity in healthy subjects by a chronic administration of a serotonin enhancer," Neuroimage, vol. 27, no. 2, pp. 299-313, 2005.

[47] C. Normann, D. Schmitz, A. Furmaier, C. Doing, and M. Bach, "Long-term plasticity of visually evoked potentials in humans is altered in major depression," Biological Psychiatry, vol. 62, no. 5, pp. 373-380, 2007.

[48] F. Chollet, J. Tardy, J. F. Albucher et al., "Fluoxetine for motor recovery after acute ischaemic stroke (FLAME): a randomised placebo-controlled trial," The Lancet Neurology, vol. 10, no. 2, pp. 123-130, 2011.

[49] A. K. Lagas, J. M. Black, W. D. Byblow et al., "Fluoxetine does not enhance visual perceptual learning and triazolam specifically impairs learning transfer," Frontiers in Human Neuroscience, vol. 10, 2016.

[50] M. H. Sharif, M. R. Talebnejad, K. Rastegar, M. R. Khalili, and M. H. Nowroozzadeh, "Oral fluoxetine in the management of amblyopic patients aged between 10 and 40 years old: a randomized clinical trial," Eye, 2019. 
[51] H. J. Huttunen, J. M. Palva, L. Lindberg et al., "Fluoxetine does not enhance the effect of perceptual learning on visual function in adults with amblyopia," Scientific Reports, vol. 8, no. 1, article 12830, 2018.

[52] P. Baumann, "Pharmacology and pharmacokinetics of citalopram and other SSRIs," International Clinical Psychopharmacology, vol. 11, pp. 5-12, 1996.

[53] J. A. Kleim, S. Chan, E. Pringle et al., "BDNF val66met polymorphism is associated with modified experience-dependent plasticity in human motor cortex," Nature Neuroscience, vol. 9, no. 6, pp. 735-737, 2006.

[54] S. T. L. Chung, R. W. Li, M. A. Silver, and D. M. Levi, "Donepezil does not enhance perceptual learning in adults with amblyopia: a pilot study," Frontiers in Neuroscience, vol. 11, p. 448, 2017.

[55] Pediatric Eye Disease Investigator Group, M. X. Repka, R. T. Kraker et al., "A randomized trial of levodopa as treatment for residual amblyopia in older children," Ophthalmology, vol. 122, no. 5, pp. 874-881, 2015.

[56] M. Epelbaum, C. Milleret, P. Buisseret, and J. L. Dufier, "The sensitive period for strabismic amblyopia in humans," Ophthalmology, vol. 100, no. 3, pp. 323-327, 1993.

[57] D. M. Levi and U. Polat, "Neural plasticity in adults with amblyopia," Proceedings of the National Academy of Sciences of the United States of America, vol. 93, no. 13, pp. 68306834, 1996.

[58] K. Bezchlibnyk-Butler, I. Aleksic, and S. H. Kennedy, "Citalopram-a review of pharmacological and clinical effects," Journal of Psychiatry and Neuroscience, vol. 25, no. 3, pp. 241-254, 2000.

[59] C. Gury and F. Cousin, "Pharmacokinetics of SSRI antidepressants: half-life and clinical applicability," Encephale, vol. 25, no. 5, pp. 470-476, 1999.

[60] M. Patris, J. M. Bouchard, T. Bougerol et al., "Citalopram versus fluoxetine: a double-blind, controlled, multicentre, phase III trial in patients with unipolar major depression treated in general practice," International Clinical Psychopharmacology, vol. 11, no. 2, pp. 129-136, 1996.

[61] E. Kucukibrahimoglu, M. Z. Saygin, M. Caliskan, O. K. Kaplan, C. Unsal, and M. Z. Goren, "The change in plasma GABA, glutamine and glutamate levels in fluoxetine- or Scitalopram-treated female patients with major depression," European Journal of Clinical Pharmacology, vol. 65, no. 6, pp. 571-577, 2009.

[62] M. J. Owens, D. L. Knight, and C. B. Nemeroff, "Second-generation SSRIs: human monoamine transporter binding profile of escitalopram and R-fluoxetine," Biological Psychiatry, vol. 50, no. 5, pp. 345-350, 2001. 


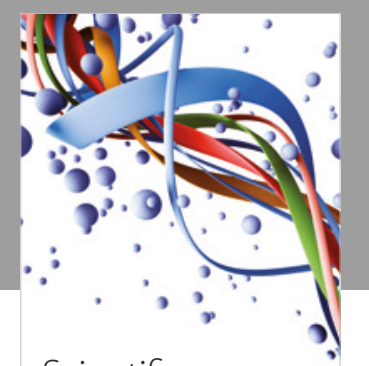

Scientifica
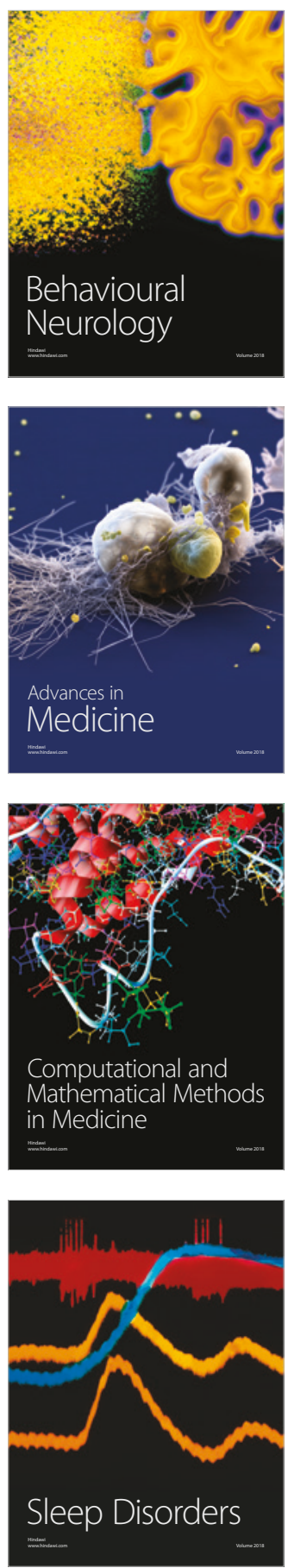

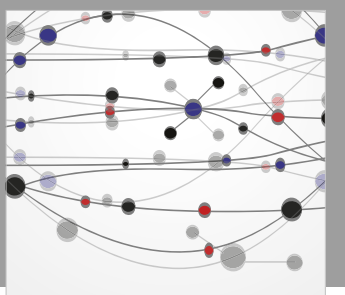

The Scientific World Journal

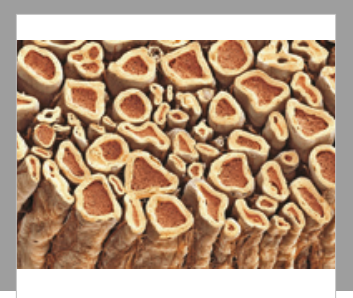

Case Reports in

Neurological Medicine

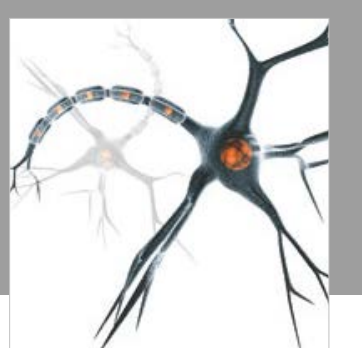

Neural Plasticity

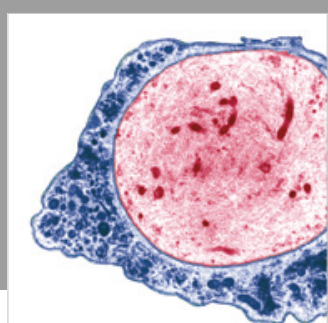

Multiple Sclerosis

International

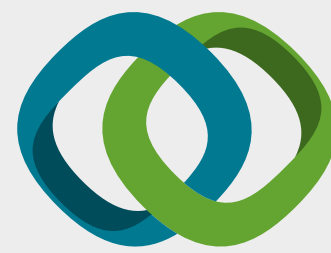

Hindawi

Submit your manuscripts at

www.hindawi.com
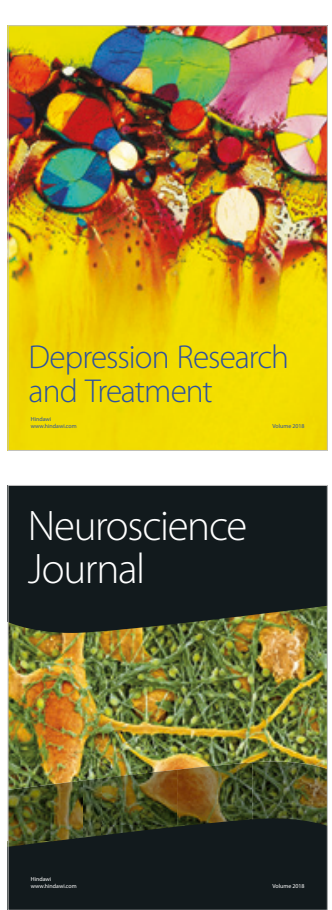

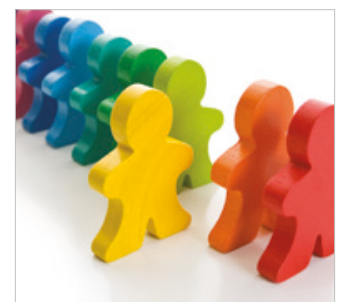

Autism

Research and Treatment
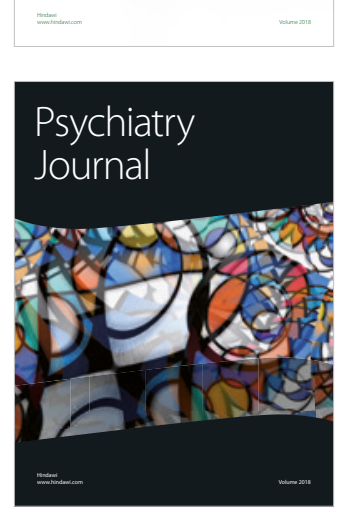
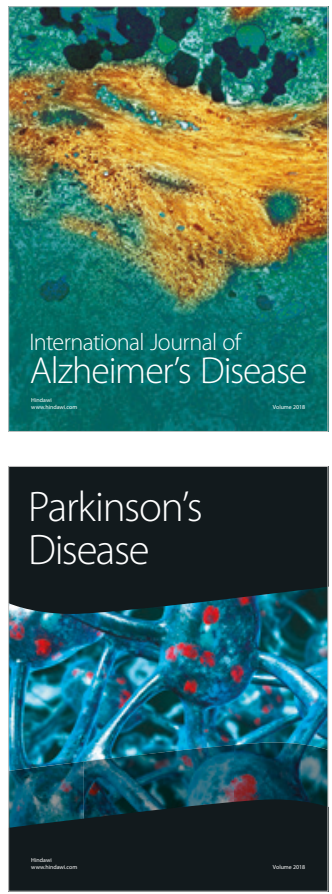
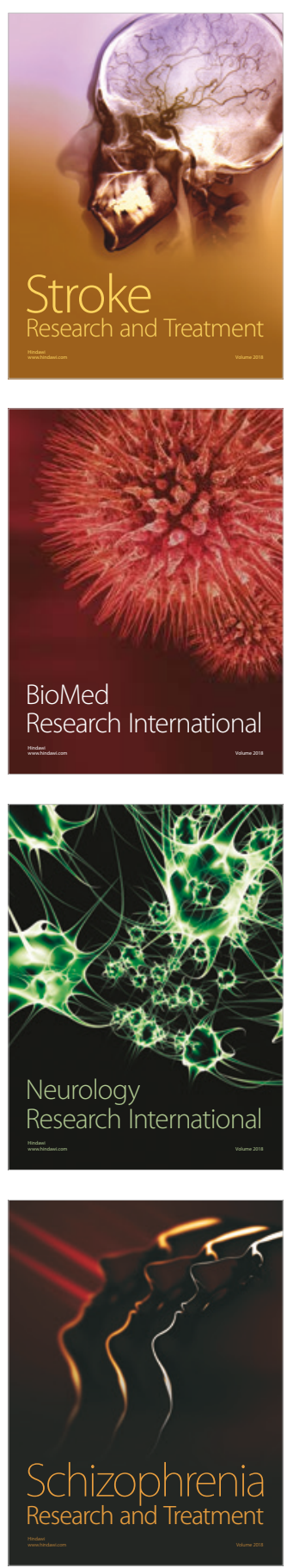